Case Report

\title{
A new support system for lateral ankle stabilization surgery: a technique guide
}

\section{Abstract}

In this article, we present a step-by-step description of a new surgical technique, which provides additional strength to the Brostrum-Gould ligament repair. The added support allows for earlier post-operative weight bearing and an earlier return to activities.

Keywords: Ankle, Ligament, Brostrum-Gould, Instability, Anchor
Volume 7 Issue 6 - 2017

Binoy Sheth,' Jaytinder S Sandhu²

'Resident Physician, Hunt Regional Medical Center, USA

IIrving Orthopedics \& Sports Medicine, USA

Correspondence: Jaytinder Sandhu, DPM Irving Orthopedics \& Sports Medicine. 2120 N. MacArthur Blvd. Irving, Texas 7506I, USA Email jaytinder.sandhu@yhoo.com

Received: March 25, 2017 | Published: April 13, 2017

\section{Introduction}

The incidence of ankle sprains has been reported to be as high as 28,000 sprains per day with ankle sprains accounting for up to $45 \%$ of all athletic injuries. ${ }^{1}$ Despite conservative treatment, activity modifications and bracing, up to $35 \%$ of patients will continue to have ankle pain, weakness and or instability resulting in Chronic Lateral Ankle Instability. ${ }^{1}$

Many surgical procedures have been described to address lateral ankle ligament pathology, including approaches that are both anatomic and non-anatomic. One of the most commonly performed anatomic repairs is the Brostrum-Gould modification. Through a midsubstance incision into the Anterior talofibular ligament (ATFL), shortening and suturing of the ligament is utilized to perform repair. To provide additional support, the Inferior Extensor Retinaculum (IER) is also incorporated into the repair.

Despite high success rates reported with this procedure, there are certain instances where this procedure is not appropriate. These include previous repair failures, ligamentous laxity, calcaneal varum and the high-demand athlete. To address these limitations, the senior author (JS) has developed an anchor-based technique to provide additional support to the lateral ankle ligament complex following repair of the ATFL.

\section{Operative technique}

The patient is placed on the operating table in the supine position. Following the administration of general anesthesia, a thigh tourniquet is applied and a bump is placed under the operative hip to internally rotate the lower extremity.

\section{Brostrum-gould}

A lateral incision is placed over the distal fibula extending to the dorsolateral foot. Blunt dissection is carried through the subcutaneous tissues down to the level of the ankle capsule (Figure 1).

The anterior talofibular ligament (ATFL) is identified, usually noted to be a thickening of the lateral joint capsule. A full thickness incision is made through the mid-portion of the ATFL. The incision curves along the anterior margin of the fibula, ending superior to the peroneal tendon sheath. Using absorbable suture, a modified Brostrum-Gould repair is performed.

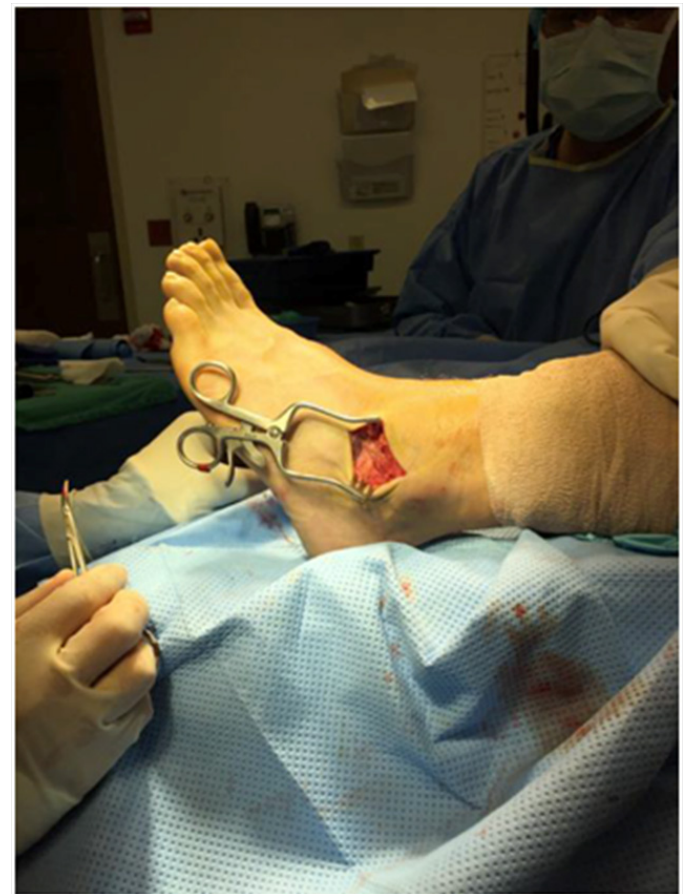

Figure I Lateral approach.

\section{Anchor placement}

\section{Talar anchor}

For placement of a $2.3 \mathrm{~mm}$ Stryker Iconix All-Suture Anchor ${ }^{\circledR}$ into the lateral aspect of the talar body, a small stab incision is made. The anchor must be placed on a non-cartilaginous surface of the lateral talus, with care not to violate the talar dome (Figure 2). A cannulated hand piece is malleted into the desired position of the anchor, distal to the normal talar attachment site of the ATFL. A drill is used to prepare the talus for anchor insertion. The anchor is placed and malleted into the talus. The suture needles are removed from the handle. Remove the handle and drill inserter. Gently, pull on all of the sutures to allow for tightening of the anchor. Cut the needles off all 4 suture tails and remove one suture tape, leaving one intact. The remaining suture tape will then be loaded into the fibular anchor (Figure 3). 


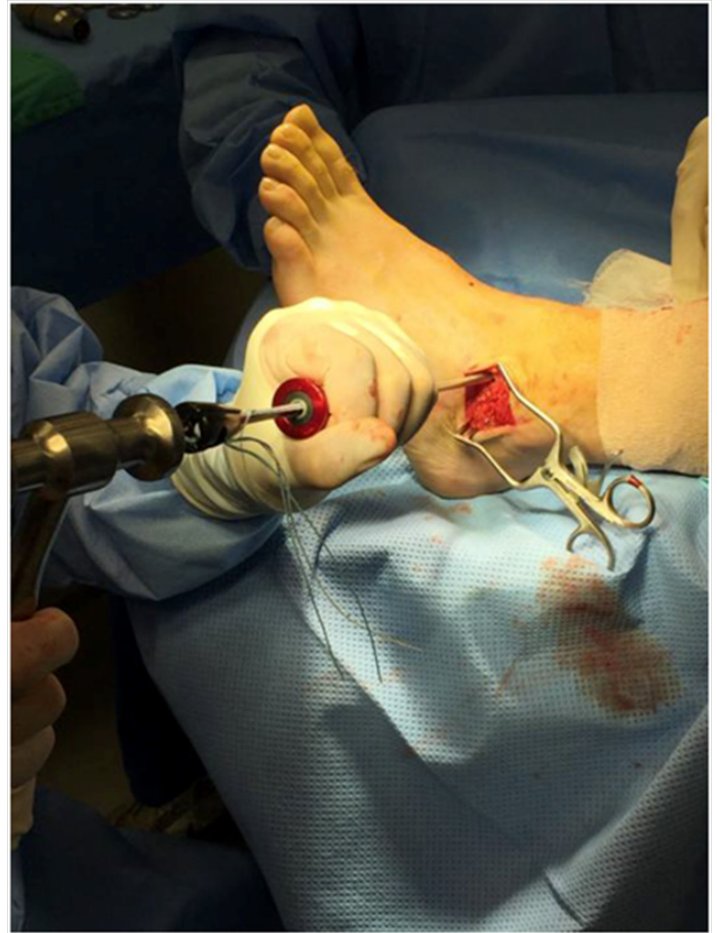

Figure 2 Placement of the Iconix anchor into the lateral talus.

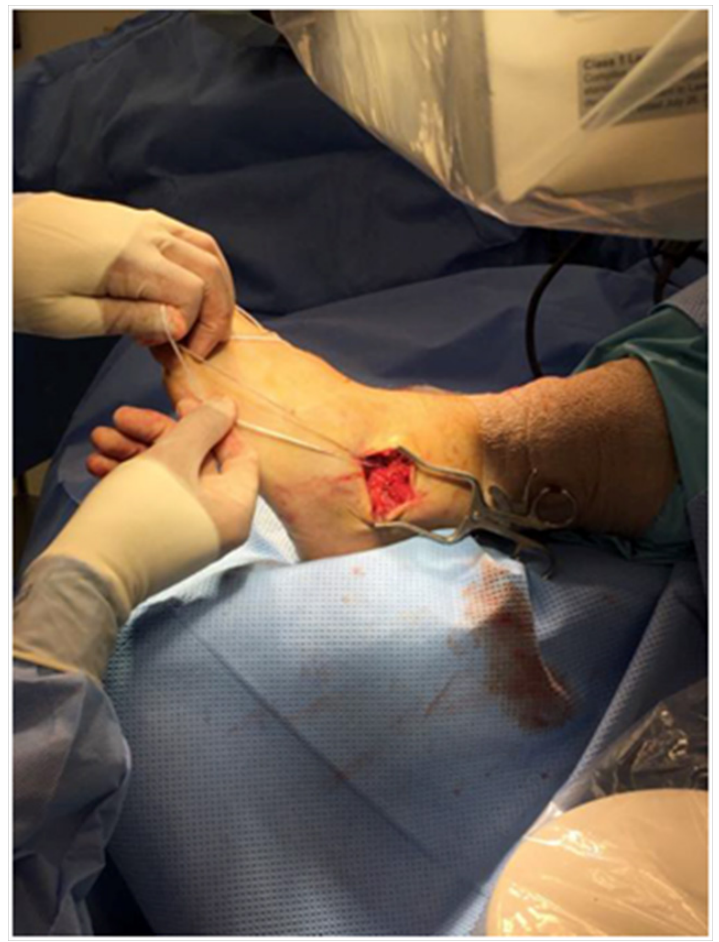

Figure 3 Following placement of the Iconix anchor into lateral talus, the suture tape ends are brought out superficially.

\section{Fibular anchor}

Expose the distal anterior surface of the fibula via a stab incision into the periosteum. A $4.0 \mathrm{~mm}$ drill is used to prepare the fibula for insertion of the ReelX STT ${ }^{\circledR}$ Anchor. It is recommended to drill just distal to the ankle joint line, aiming for the central aspect of the fibula and avoiding the distal tip. Drill anterior to posterior. The suture tails from the talar anchor are then fed into the islets of the ReelX anchor, 1 tail per islet. Ensure that the suture tails are of equal length. The anchor is placed and malleted into the fibula (Figure 4). The suture tape is placed in an extra-capsular manner and oriented parallel to the ATFL. The foot is then placed and held in a dorsiflexed and everted position. The black knob of the yellow handle is slowly rotated clockwise, which advances and tightens the suture tape (Figure 5). It is recommended to place a hemostat under the suture tails, to continuously assess the tension.

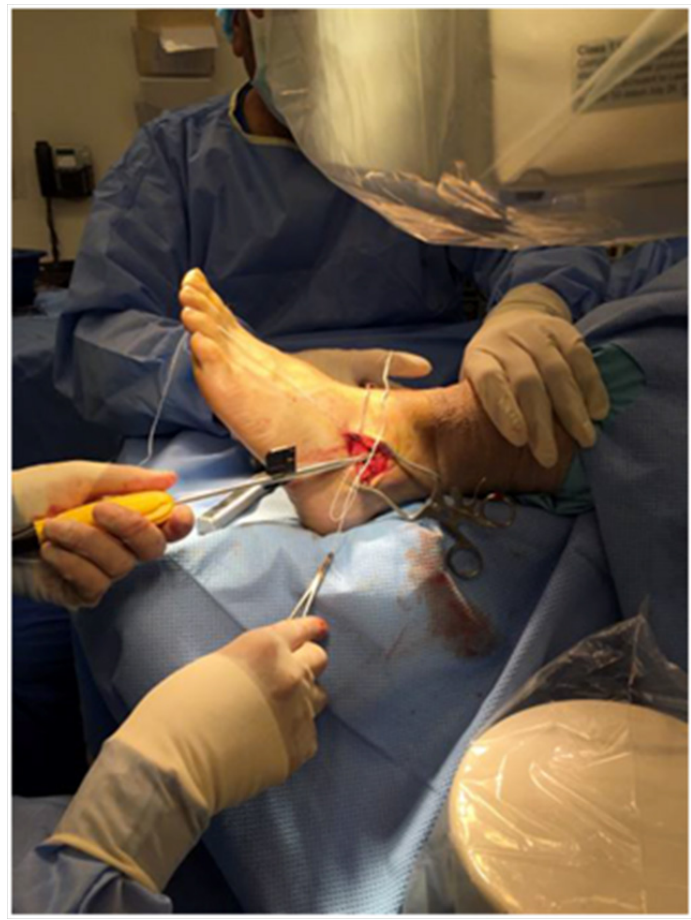

Figure 4 Placement of the ReelX STT $®$ anchor into the distal fibula.

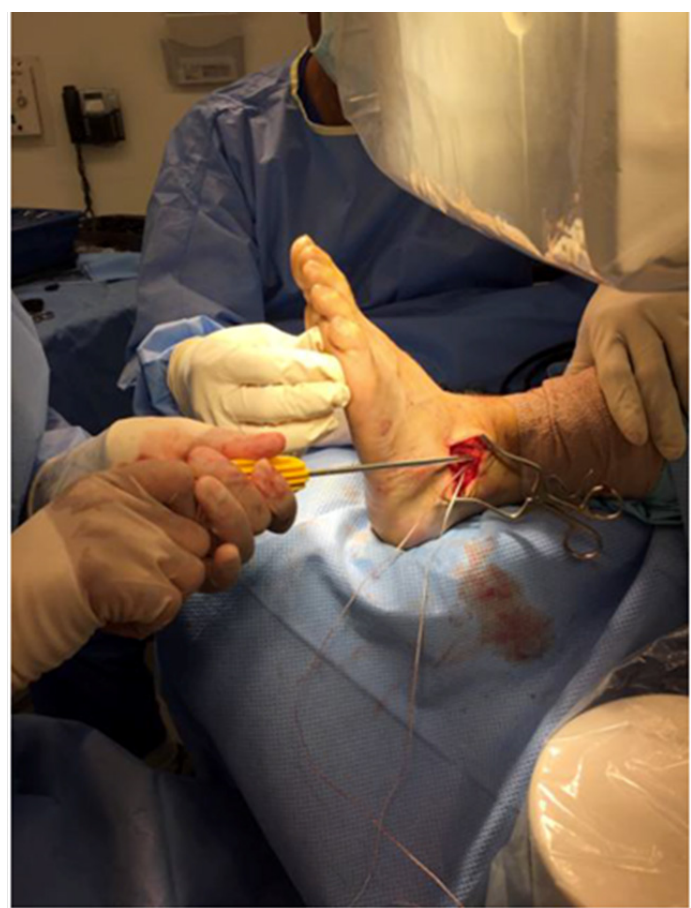

Figure 5 With the foot held in dorsiflexion and eversion, clockwise rotation of the black knob is performed to advance the suture tape, which applies additional strength to the repair site. 
Continue slowly rotating the black knob until the desired amount of tension is achieved. Do not over tighten the suture tape. The handle is then removed and a 15-blade is used to cut the suture tape. Ankle joint range of motion, via the anterior drawer test, is repeated to verify stability of the repair and anchors (Figure 6).

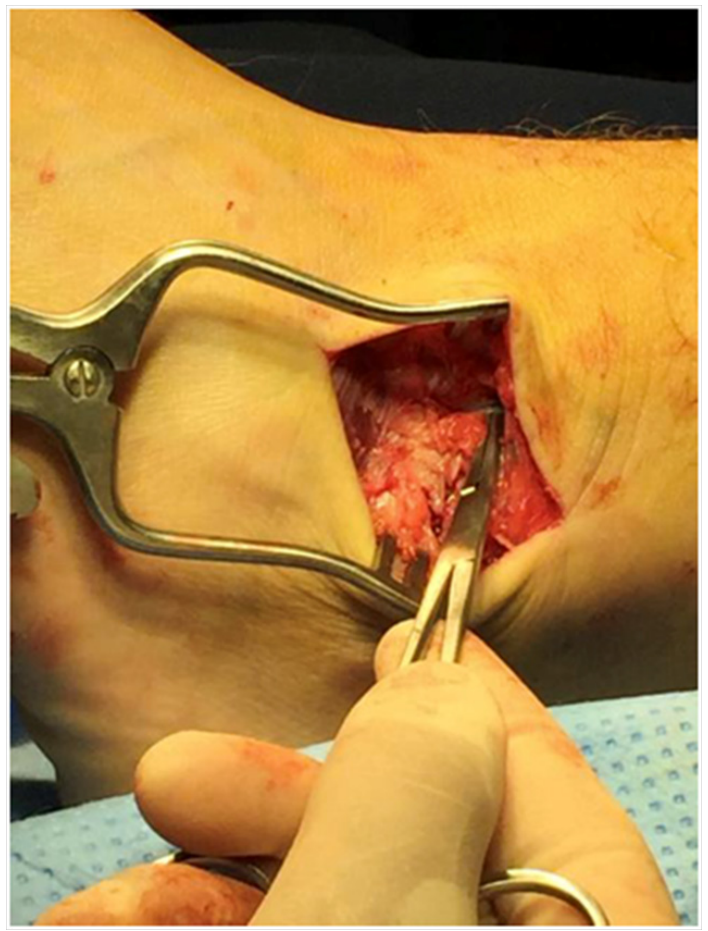

Figure 6 Final image of the extra-capsular, tensioned suture tape placed parallel to ATFL.

Intraoperative images may be taken to verify the position of the Reelx anchor within the fibula, however the Iconix anchor is not visible on $\mathrm{x}$-ray (Figure 7).

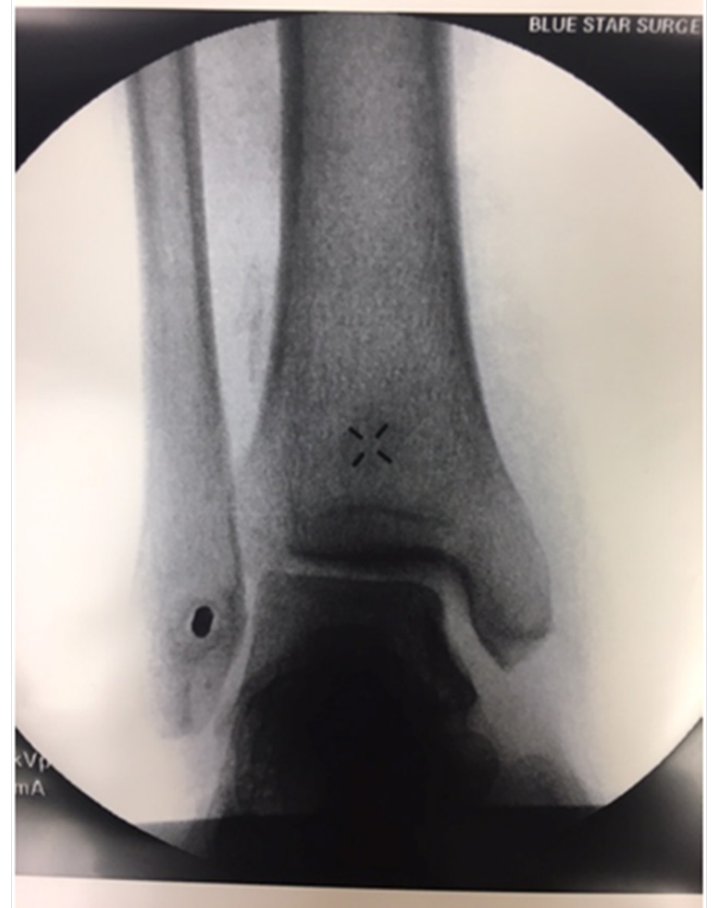

Figure 7 Intra-operative film showing the position of the ReelX STT $®$ Anchor in the distal fibula.
Layered closure is then performed, and a well-padded posterior splint is applied with the foot in a dorsiflexed and everted position.

The post-operative course is dependent on concomitant procedures performed. When performed as an isolated procedure, the patient is placed in a non-weight bearing posterior splint for 2 weeks. Following suture removal, the patient is permitted full weight bearing in a removable pneumatic walker boot and physical therapy begins. 4 weeks post-operatively, the boot is removed and the patient is able to transition into supportive shoe gear, avoiding flip slops and sandals. A lace up ankle brace may be used at this time as needed. Return to normal activities is generally permitted at $8-10$ weeks, when the patient is clinically able to perform the single limb stand and single limb hop tests without any pain or weakness.

\section{Discussion}

Long-term follow up studies of the Brostrum-Gould have shown success rates and patient satisfaction ranging from to $87-95 \% 2$. This popular ligament repair does, however, have a number of limitations and contraindications- including being overweight, having a cavus foot type, and being a high-demand worker or athlete. ${ }^{2}$ A number of surgical techniques have been reported in the literature over the years to provide additional strength to repaired lateral ankle ligaments, which include the use of one or more anchors.

The ReelX STT ${ }^{\circledR}$ anchor (Stryker Corporation, Kalamazoo, Michigan) we utilize in the fibula is unique as suture can be loaded into it and the desired amount of tension can be precisely applied by twisting the proximal end of the inserter device. The final amount of desired tension placed on the suture tape is maintained by the anchor's construct. Studies have reported that 60 degrees of clockwise rotation will allow for $1.5 \mathrm{~mm}$ of suture advancement. ${ }^{3}$ When the fixation strength of the ReelX STT ${ }^{\circledR}$ anchor was compared to six competitive knotless anchors, ReelX STT ${ }^{\circledR}$ was found to reach the highest load at $3 \mathrm{~mm}$ of displacement (126N) and the highest ultimate load (302 N)3. To our knowledge, this is the first reported use of such an anchor in lateral ankle ligament surgery.

\section{Conclusion}

The authors present a new technique in lateral ankle stabilization surgery which reinforces the Brostrum-Gould ligament repair. Though future studies are needed, we feel this procedure will provide greater strength when compared to ligament repairs alone. Similar techniques have been popularized and presented in recent years, however our system only requires two anchors per ligament repair, which decreases operative time, materials, and cost. In addition, no other previously reported system or technique allows for direct and precise tightening of the suture tape through a specially designed anchor.

While not indicated for all ankle ligament repair surgeries, the authors encourage foot and ankle surgeons to consider this support system in situations when the primary repair of lateral ankle ligaments is not strong enough to achieve the desired support.

\section{Acknowledgements}

The authors would like to thank Mr. Kyle Carey, Stryker Sports Medicine, for his help and support.

\section{Disclosure}

The senior author (JS) is a consultant for Stryker, Endoscopy.

\section{Conflicts of interest}

None. 


\section{References}

1. Adams J, Barton E, Collings J et al. Emergency Medicine. Saunders, Elsevier, Philadelphia, USA, pp. 2008;897-898.
2. O'Brien T, Baumhauer JF Surgical Considerations in the Treatment of Ankle Instability.J Athl Train 2002;37(4): 458-462.

3. Stryker Evidence Matters Research Bulletin Tech Report \#RD-10-036. ORS. 2011. 